\title{
Care load and affecting factors of alzheimer patients
}

\begin{abstract}
Alzheimer's is one of diseases, the incidence increases with age. The disease, which even destroys even the ability to perform simple tasks, has no treatment options, so it requires care. Caregivers may have difficulty with the burden of care and may feel lonely, helpless and unhappy. Therefore, the psychological well-being of caregivers has recently become one of the most interesting research topics. Caregivers are not enough knowledgeable about Alzheimer's disease and how to approach their patients. For caregivers to be healthier and to provide healthier care; should benefit care, support and treatment services. Appropriate care training should be provided to reduce the burdens of caregivers; necessary measures should be taken to protect their social life and psychological health.
\end{abstract}

Keywords: alzheimer, burden of care, caregivers, public health, neurology, giving care
Volume 3 Issue 6 - 2019

\author{
Ferhan Soyuer \\ Department of Physiotherapy and Rehabilitation, Faculty of \\ Health Sciences, Nuh Naci Yazgan University, Turkey
}

\begin{abstract}
Correspondence: Ferhan Soyuer, Department of Physiotherapy and Rehabilitation, Faculty of Health Sciences, Nuh Naci Yazgan University, Turkey, Tel 054223540 62, Email soyuerferha@gmail.com
\end{abstract}

Received: November 24, 2019 | Published: December 16, 2019
Abbreviations: AD, alzheimer's disease; DSM-IV, association diagnostic and statistical manual of mental disorders

\section{Introduction}

Today, the increase in the elderly population in the world population is remarkable. This increase in the elderly population also causes an increase in the prevalence of diseases related to old age. One of these diseases, Alzheimer's Disease (AD) is increasingly becoming one of the most important problems of public health and neurology. ${ }^{1,2}$ The incidence of $\mathrm{AD}$ increases with age (AD is seen in 8 out of 100 people over 65 years of age). In the coming years, will the incidence of the disease appears to increase further. ${ }^{1,2}$ In the American Psychiatric Association Diagnostic and Statistical Manual of Mental Disorders (DSM-IV), AD is defined as the presence of many cognitive disorders including memory destruction without deterioration in consciousness. $^{3}$ The National Institute of Aging includes Alzheimer's Disease; It defines a progressive, irreversible brain disorder that slowly destroys memory and thinking skills and even the ability to perform simple tasks. ${ }^{4}$ Since there is currently no treatment option that prevents or stops the development of $\mathrm{AD}$, the target is to improve and support the quality of life of patients, relatives and caregivers as much as possible. As the stages of AD progress, the symptoms of the disease are exacerbated. In daily living activities, the dependence on caregivers increases and the presence of a caregiver becomes indispensable. The care of these patients; It is known that there is a society problem too including their caregivers and their families. ${ }^{4,5}$

The annual costs of AD are stated to be $\$ 100$ billion on average. 61 billion dollars of this figure consists of professional caregivers fees and nursing homes expenses. The loss of workforce at home caregivers for Alzheimer's patients was determined as 10.2 billion dollars annually. 5,6 There are not enough planned nursing homes, day hospitals or nursing homes for AD. Generally, elderly patients are cared for by their first-degree relatives and at home. AD care; it is generally carried out by spouses, children, other relatives, friends or neighbors. ${ }^{5}$ Caregiving often remains on the shoulders of a person and most the time can be forced against the care load, oneself may feel lonely, hopeless and unhappy. The psychological well-being states of caregivers has recently become one of the most interesting research topics.

\section{What is maintenance?}

Giving care; It is defined as a task that consumes time and energy and is materially and physically exhausting. Maintenance service; not limited to a single aid; it covers health care (medication, treatment, monitoring, etc.), personal care (bathing, nutrition, toileting, dressing, etc.), coordinating the social services of the patient, shopping and home management, financial assistance and sharing the same house. ${ }^{5,6}$ Giving care; As formal care and informal care are divided into 2:

1.Formal care; While it covers professional care provided by private and public institutions,

2.Informal care; By spouses, by children, by friends and relatives can be defined as a whole of services that are provided without charge and include non-professional care. Family members caring for AD are also included in informal care. It was found that $37.9 \%$ of the caregivers were the bride of the patient, $33.9 \%$ were the patient's children, $10.7 \%$ were the patient's spouses, $10.2 \%$ were the siblings, and $7.3 \%$ were the caregivers and groom's..$^{5-7}$ Especially for AD, caregiving involves a very long and tiring process. In this process, caregiving family members are facing many psychological, physical, economic and social problems and they are under be influenced by care burden.

\section{What is maintenance burden?}

Care burden is defined as a multidimensional response to physical, psychological, social and financial stress factors associated with caregiving experience. The care burden results from the caregiver perceiving these effects and stress factors. Therefore maintenance burden; influenced by psychosocial factors such as social environment, culture and degree of kinship.

I. Caregiving are separated formal care into as informal care.

II. Causes such as emotional commitment, empathy and love that require thinking of others,

III. The desire to prevent the guilt that he may experience, when he does nothing,

IV. The desire of the currently dependent elderly to be indebted for 
what they have done in the past or to pay their debt by providing help and support,

V. The belief that the elderly can not be able to meet their needs due to of their 'patient role',

VI. Because of the social values such family solidarity and responsibility for being a child, the responsibility for the care of the elderly undertaken,

VII. The use of the financial means of the elderly can be considered among the reasons to care for the elderly. ${ }^{6,7}$

\section{The factors that shape the burden of care in alzheimer's disease}

It is stated that there are many factors that shape the psychological problems and care burden experienced by caregivers. Caregiving is a multi-foot process. Among the Factors Shaping Care Burden in AD;

I. Patient belong: personal properties such as age, gender

II. Disease characteristics, disease's level

III. Caregiving features

IV. The Careviger's age, gender, ethnicity, educational status, health, working status, occupation, economic status, culture level, degree of intimacy with the patient and relationship with patient, whether voluntary in care, the average of hours of care provided per day, as well as whether the caregiver takes support or not, whether they sharing the same house with the elderly are among the factors affecting the burden of care.

V. Environmental conditions are counted.

\section{Patient characteristics}

The fact that the patient's age was younger and that the patient's gender was male was considered to be rated as least significant important in the care burden. In the care burden, cognitive deficiencies (dimensions of forgetfulness and loss of high cortical functions), functional deficiencies (auxiliary daily living activities and inability to do daily living activities, the number and violence of behavioral and psychiatric symptoms (agitation, delusion, hallucinations) are stated to be effective. ${ }^{7,8}$

\section{Disease characteristics}

Early Stage AD is the stage in which the caregiver recognizes the disease and the patient's diagnosed. In this phase, the caregiver usually focuses on diagnosis, understanding the disease and future plans Intermediate phase and advanced stage $\mathrm{AD}$, are periods in which the caregiver and the patient need permanent support and assistance. In these phases, caregivers; they have to cope with issues such as medical care and treatment of the patient, patient safety, patient care support and at last period patient care.

\section{Daily activities}

ADL; It was suggested that the caregiver is an indicator of how busy the with giving care is, and how much help the provides for $\mathrm{ADL}$ is as intensive as the caregiver. It was reported that caregivers of Alzheimer's Patients are doing more to help patients in their daily living activities compared to caregivers of other elderly patients.

\section{Psychiatric symptoms}

Caregivers evaluated the most difficult symptoms of Alzheimer's disease as delusions, hallucinations, depression, and physical aggression. In the studies, stress rate was found to be $64 \%$ in the caregivers of $\mathrm{AD}$ with delusions, while this rate was found to be $33 \%$ in caregivers without delusions. However, there are some who argue that the changes in the cognitive abilities and behaviors of the patients do not affect all caregiver to the same extent, and instead is closely related to the subjective perception of social support and caregiving and the feeling of burden. With questions and signs of behavior such as tapping; psychological symptoms such as delirium, anxiety; It is stated that patient, familys and caregiver increase their distress even further and therefore they may cause early placement in the nursing home, increase the cost of care and decrease the quality of life. ${ }^{7,8}$ Elderly and / or spouse caregivers that they are better know of the patient's premorbid personality and behaviors; environmental factors, interactions with the patient, they put forward that they can regulate in a way to prevent behavioral disorders. A significant relationship was found between the behavioral and psychological symptoms of the patient and loss of appetite, eating disorders, aggression, agitation, irritability and care burden.

\section{Depression caregivers of alzheimer's patients}

In the studies showing that the caregivers of Alzheimer's patients experience a heavy burden during at the care process, psychological stress and long period and try to cope with emotional problems; When compared with the control groups of the same age, it was seen that those who caregiving for Alzheimer's patients have a much higher physical and psychological morbidity than others. ${ }^{8,9}$ While the prevalence of psychiatric symptoms was found to be $52 \%$ in Alzheimer patients' caregivers, this rate was found to be $15-20 \%$ in the general population..$^{10}$ It was reported that caregivers of Alzheimer's patients experience higher levels of psychological stress compared to caregivers of patients with other chronic disease diagnoses ${ }^{8,9}$ In most studies, it was reported that $10-83 \%$ of those caring for Alzheimer's patients were diagnosed with major depression at clinical level. ${ }^{8,9}$

In caregivers of Alzheimer's patients, at more than $50 \%$ of the patients within one year after diagnosis of the disease; newly diagnosed, moderate-severe depression was detected. The risk of depression in caregivers of Alzheimer's patients; caregiver age, gender, physical skills, personality and social support level,worsening health situtiation and was found to be associated with behavioral impairment in patients. ${ }^{11,12}$ Depression; It is one of the negative results of the caregiving process, which decreases the quality of life of caregivers, causes them to deteriorate functionally and causes an increase in mortality emotional and physical burden in care; It causes caregivers to experience more anxiety and depression, impair their health, go to more doctors, and use more psychotropic drugs. It has been shown that $49 \%$ of female caregivers and $31 \%$ of male caregivers experience depression in Alzheimer's patients, and 50-52\% of female spouses and $21-25 \%$ of male spouses develop depression. ${ }^{11,12}$

\section{The diseases}

It has also been shown that caring for Alzheimer's patients has a markedly effect on the physical health. Sadness and poor health habits increase the level of stress hormones, which in turn activate physiological processes leading to adverse health outcomes such as hyperglycemia, hyperinsulinemia, hypertension and weakened 
immune response. These statuses progress, deteriorate the health of the person and reveal diseases. As a result of the examination of the caregivers according to the non-caregivers; It was found that their health status was worse, they used more drugs, their stress hormone levels were $23 \%$ higher and their antibody responses were $15 \%$ lower.

\section{Caregiving features}

It is stated that who giving cares for the patient, how many hours a day at cared for, how many months / years he/she has cared to the patient, whether he/she has received help, his/her experience and skills in the of caregiver-patient care process and the psychological problems experienced by caregivers shape the burden of care. In the USA, it was found that $15 \%$ of those who maintenance for people with Alzheimer's disease care more than 40 hours per week. As the stage of the disease progresses, the hour of care increases. Some caregivers living with Alzheimer's patients stated that they care for the patient 24 hours a day, seven days a week, sleep with the patient at night and have to assist with all daily living activities. ${ }^{12,13}$ Physical limitations of $\mathrm{AD}$, behavioral problems and long care hours; on the spouses has brought more care burden than adults-children. The reason for this status, it is generally thought that caregivers spouse have their own chronic diseases and age-related problems.

Giving care; it can be more challenging for caregivers if the caregiver has career and there are another family members to care for. Due to the absence of other caregivers or assistants and depending on sharing the same home with the patient, caregiver, it has been reported that it causes prolongation of the time it spends on giving care and increases the burden of care and lowers the well-being. ${ }^{13,14}$ The load level's of male or female spouses, girls or boys living with Alzheimer's patients increased and mental health deteriorated gradually. However, this situation was found mostly in girls. In those living with $\mathrm{AD}$, feeling tired and stressed were $60 \%$, depression $48 \%$, hopelessness $28 \%$ and suicidal ideation $16 \%$ has been identified. These rates much lower in those who did not live together. ${ }^{13,14}$

\section{Demographic characteristics of patient and caregiver \\ Age}

There are not many studies on this subject. We know that; The caregivers of $\mathrm{AD}$ are usually middle-aged and older. On the other hand, some studies show that elderly caregivers perceive less care burden than younger caregivers and that a being young age was a predictor of caregiver depression. It was stated that young caregiver is more affected negatively from the care process and the burden level increases as the caregiver age decreases. Because young people are at the beginning of their careers, they may have more problems when faced with a heavy burden of care. ${ }^{14,15}$

\section{Gender}

It is stated that family care responsibility of Alzheimer's patients is undertaken by women at a rate of $75-80 \%$, usually one person takes all responsibility and it is not equal sharing between family members. The responsibility of maintenance is primarily; patient's wife, daughter (single or grand daughter) and patient's bride; if there is no family member, it is seen that other relatives, kin, neighbors or friends take over. It can be seen at female caregivers have more anxiety, depression and emotional stress than male caregivers. The reason for this can be explained in different ways such as the fact that the prevalence of depression in women is already high, and that women have more responsibilities in society, they become mother and earn money for livelihood, they manage household chores and assume emotional supportive roles. ${ }^{14,15}$

\section{Education status}

In general, there are studies that say educated caregivers are more likely to have many life skills such as communication skills, stress management techniques. It is stated that educated people may have more financial resources and in this case they can find ways to cope without stress symptoms. ${ }^{16,17}$ Caregivers at lower educational levels are more likely to experience burden, as the risk of developing stress symptoms is likely to be higher. ${ }^{17,18}$

\section{Working status}

It has been seen that caregivers have had to quit their jobs or reduce the time they devote to their work to a great extent because of their caregiving responsibilities. It is stated that while working caregivers are trying to maintain not only business life but also family life, they have to do the third shift for the patient and they cannot allocate enough time for themselves. In a study that did not include men, it was shown that working women felt intense pressure both because they continued their caregiving work and their work. There was no significant difference in depressive symptoms between working caregiving and who were inoperative women caregivers. However, it has been found that both woman who work and giving care, whether or not they work, show more depressive symptoms than women who do not giving care. ${ }^{19,20}$ from the women who giving care, the more active in their social environment feel better; less social ones have a higher rate of depressive symptoms. ${ }^{20}$

\section{Economical status}

In addition to spending a lot of time on giving care activities, it has been shown to affect caregivers as economically. Caregivers with poor economic status find forced to provide services, tools and medical treatment to assist in the care work. It is suggested that those with less financial problems have wider opportunities to meet social support services. ${ }^{21,22}$

\section{Environmental conditions at giving care}

It has been suggested that factors such as the number of rooms of the house, the adequacy of the bathroom, the status of the elevator or stairs may affect the maintenance load. It has been identified that the caregivers, thought that received little support from their environment increased the care burden. Especially incoming support from the family; it is stated that the patient affects the care load more priority than the disease state..$^{22,23}$

\section{Effects of stages of alzheimer's disease on caregiver}

\section{A. Early stage alzheimer's disease}

This first stage of acquaintance of the patient's relatives with the disease may cause a crisis situation that suddenly affects the family system and relations. Usually the first thing to know is the cause of the disease and how it will proceed. Physical and emotional experiences, fear of the patient loss, changing roles in the family and economic difficulties come with it. This is a process that affects both the patient diagnosed with $\mathrm{AD}$, her/him family members who giving care for her/ him, and even her distant relatives and friends. In the long period, problems such as evident difficulties caused by behavioral changes, 
complete memory loss, social consequences formed by their,material burdens may arise. The relationships between spouses, children, grandchildren with, the ill member of the family is impaired and difficulties in accepting this diagnosis are experienced. The wife of the patient loses her life partner, the kids lose their parents who now turned into total strangers, parents who taught and guided them their lives. The pain of these losses is often not revealed enough, however it stays a heavy psychological stress that the family must cope with. ${ }^{23,24}$

\section{B. Middle stage alzheimer's disease}

In addition to cognitive problems, it was stated that the caregiver was under more burden in the middle stage, which included physical and psychiatric problems. From the middle stage, AD can often show mismatch in issues such as sleeping, eating, bathing or dressing, and hence, caregivers can live more difficulty. It is clear that as the severity of the disease increases and as secondary problems are added to the disease, this difficulty will increase further. In $\mathrm{AD}$, with the progression of stages, with increased dependence in activities of daily living, caregiver burden is also stated to increase..$^{24,25}$ In patients, it has been identified that physical aggression to caregivers was more common in the middle stage $(\% 34)$ than in the early stage $(\% 4)$. The caregiver was found to have the highest emotional complexity and sadness in the middle stage and increased linearly as the stages progressed.

\section{Advanced stage alzheimer's disease}

The most important problems in the advanced stage are the difficulties of dealing with the patient who needs continuous care, the increase in medical problems, the difficulties of reaching helplessness such as nutrition, mobilization or drug use. The care of the patient, who has become bed-dependent, is a separate specialty. In the advanced stage, communication problems begin to make itself felt more. Making sure that the patient hears, making simple sentences while talking, adjusting the tone; in order to the patient understand; gesture and facial expressions, efforts to provide communication using hand,arm and body language are the tasks of the caregiver's at this stage. It was stated that the caregiver children of the patient, tend to place Alzheimer's patients in a nursing home and feel emotionally comfortable. This reduced their intense difficulty in the middle stage and took care loads.On the other hand, it was found that co-caregivers had the highest level of sadness at this stage. In caregivers, for reduce burnout; exercise, physical activity, meditation programs, hypnosis, individual and group therapy and relaxation exercises benefit can be seen. With these methods, caregivers have been shown to decrease depression, anxiety rates and frequencies of medicine use. It has been reported that family visits can be an effective method that reduces caregiver burnout and that other members of the family can effective take action to provide support and responsibility to the caregiver. ${ }^{24,25}$

\section{Conclusion}

Caregivers of Alzheimer's patients; they can't reach the psychological, economic and social resources they need. Caregivers state that they do not know about Alzheimer's disease and how they should approach their patients and they are note they want to be informed about it. Spread of caregiving course, participation of caregivers in courses, or experts' to be appointed by the authorities, support for themselves in their homes is among the expectations. In the 2011 report of the World Health Organization, it was recommended that each country should have a health strategy that focuses on the early diagnosis of with caring on the alzheimer disease knowledge' of health workers and caregivers . For Alzheimer patients' relatives to be healthier and offer a healthier care; at their should benefit from with care, support and treatment. The way of decreasing the amount of tension on the care taker lies in taking necessary precautions in protecting the psychological health and social life of the care taker as well as educating the care taker with appropriate education.

\section{Acknowledgements}

None.

\section{Conflcits of interest}

The author declares there is no conflict of interest.

\section{References}

1. Lane CA, Hardy J, Schott JM. Alzheimer's disease. Eur J Neurol 2018;25(1):59-70.

2. Chazot-Balcon M, Bouchard JP. Caring for people suffering from Alzheimer's disease. Rev Infirm. 2019;68(247):32-34.

3. Carl C. DSM-IV: Diagnostic and statistical manual of mental Disorders. JAMA. 1994;272(10):828-859.

4. Hyman BT, Phelps CH, Beach TG, et al. National Institute on AgingAlzheimer's Association guidelines for the neuropathologic assessment of Alzheimer's disease. Alzheimers Dement. 2012;8(1):1-13.

5. Takizawa C, Thompson PL, van Walsem A. Epidemiological and economic burden of Alzheimer's disease: a systematic literature review of data across Europe and the United States of America. J Alzheimers Dis. 2015;43(4):1271-1284.

6. Dawood S. Caregiver Burden, Quality of Life and Vulnerability Towards Psychopathology in Caregivers of Patients with Dementia/Alzheimer's Disease. J Coll Physicians Surg Pak. 2016v; 26(11):892-895.

7. Alzheimer's Association. 2018 Alzheimer's disease facts and figures. Alzheimers Dement. 2018;14:367-429.

8. Liu S, Li C, Shi Z, et al. Caregiver burden and prevalence of depression, anxiety and sleep disturbances in Alzheimer's disease caregivers in China. J Clin Nurs. 2017;26(9-10):1291-1300.

9. Lou Q, Liu S, Huo YR. Comprehensive analysis of patient and caregiver predictors for caregiver burden, anxiety and depression in Alzheimer's disease. J Clin Nurs. 2015;24(17-18):2668-2678.

10. Linden M, Linden U, Schwantes U. Disability and functional burden of disease because of mental in comparison to somatic disorders in general practice patients. Eur Psychiatry. 2015;30(6):789-792.

11. Dawood S. Caregiver burden, quality of life and vulnerability towards psychopathology in Caregivers of Patients with Dementia/Alzheimer's Disease. J Coll Physicians Surg Pak. 2016;26(11):892-895.

12. Chen CT, Chang CC, Chang WN. Neuropsychiatric symptoms in Alzheimer's disease: associations with caregiver burden and treatment outcomes. QJM. 2017;110(9):565-570.

13. Monteiro AMF, Santos RL, Kimura N. Coping strategies among caregivers of people with Alzheimer disease: a systematic review. Trends Psychiatry Psychother. 2018;40(3):258-268.

14. Yang Z, Levey A. Gender differences: A lifetime analysis of the economic burden of Alzheimer's disease. Women Health Iss. 2015;25(5):436-440.

15. Kimura NRS, Baptista MAT, Santos R. Caregivers' perspectives of quality of life of people with young- and late-onset Alzheimer Disease. $J$ Geriatr Psychiatry Neurol. 2018;31(2):76-83. 
16. Clay E, Zhou J, Yi ZM, et al. Economic burden for Alzheimer's disease in China from 2010 to 2050: a modelling study. J Mark Access Health Policy. 2019;7(1):1667195.

17. Garmon C. Understanding the importance of the caregiver in the Alzheimer's patient's plan of care. J Natl Black Nurses Assoc. 2019;30(1):40-47.

18. Szabo S, Lakzadeh $\mathrm{P}$, Cline $\mathrm{S}$. The clinical and economic burden among caregivers of patients with Alzheimer's disease in Canada. Int J Geriatr Psychiatry. 2019;34(11):1677-1688.

19. Schumann C, Alexopoulos P, Perneczky R. Determinants of self- and carer-rated quality of life and caregiver burden in Alzheimer disease. Int J Geriatr Psychiatry. 2019;34(10):1378-1385.

20. Suehs BT, Davis CD, Alvir J, et al. The clinical and economic burden of newly diagnosed Alzheimer's disease in a Medicare Advantage population. Am J Alzheimers Dis Other Dement. 2013;28(4):384-392.
21. Kirson NY, Desai U, Ristovska L, et al. Assessing the economic burden of Alzheimer's disease patients first diagnosed by specialists. BMC Geriatric. 2016;16:138.

22. Zucchella C, Bartolo M, Pasotti C. Caregiver burden and coping in earlystage Alzheimer disease. Alzheimer Dis Assoc Disord. 2012;26(1):55-60.

23. Hirono $\mathrm{N}$, Tsukamoto $\mathrm{N}$, Inoue $\mathrm{M}$, et al. Predictors of long-term institutionalization in patients with Alzheimer's disease: role of caregiver burden. No To Shinkei. 2002;54(9):812-818.

24. Chiatti C, Furneri G, Rimland JM. The economic impact of moderate stage Alzheimer's disease in Italy: evidence from the UP-TECH randomized trial. Int Psychogeriatr. 2015;27(9):1563-1572.

25. Michaud TL, High R, Charlton ME, et al. Dependence stage and pharmacoeconomic outcomes in patients with alzheimer disease. Alzheimer Dis Assoc Disord. 2017;31(3):209-217. 